This item was submitted to Loughborough's Research Repository by the author.

Items in Figshare are protected by copyright, with all rights reserved, unless otherwise indicated.

\title{
Modeling the sputter deposition of thin film photovoltaics using long time scale dynamics techniques
}

PLEASE CITE THE PUBLISHED VERSION

http://dx.doi.org/10.1557/opl.2011.1124

\section{PUBLISHER}

Cambridge University Press (c) Materials Research Society

VERSION

AM (Accepted Manuscript)

LICENCE

CC BY-NC-ND 4.0

\section{REPOSITORY RECORD}

Blackwell, Sabrina, Roger Smith, Steven D. Kenny, and Michael Walls. 2019. "Modeling the Sputter Deposition of Thin Film Photovoltaics Using Long Time Scale Dynamics Techniques”. figshare. https://hdl.handle.net/2134/11621. 
This item was submitted to Loughborough's Institutional Repository (https://dspace.lboro.ac.uk/) by the author and is made available under the following Creative Commons Licence conditions.

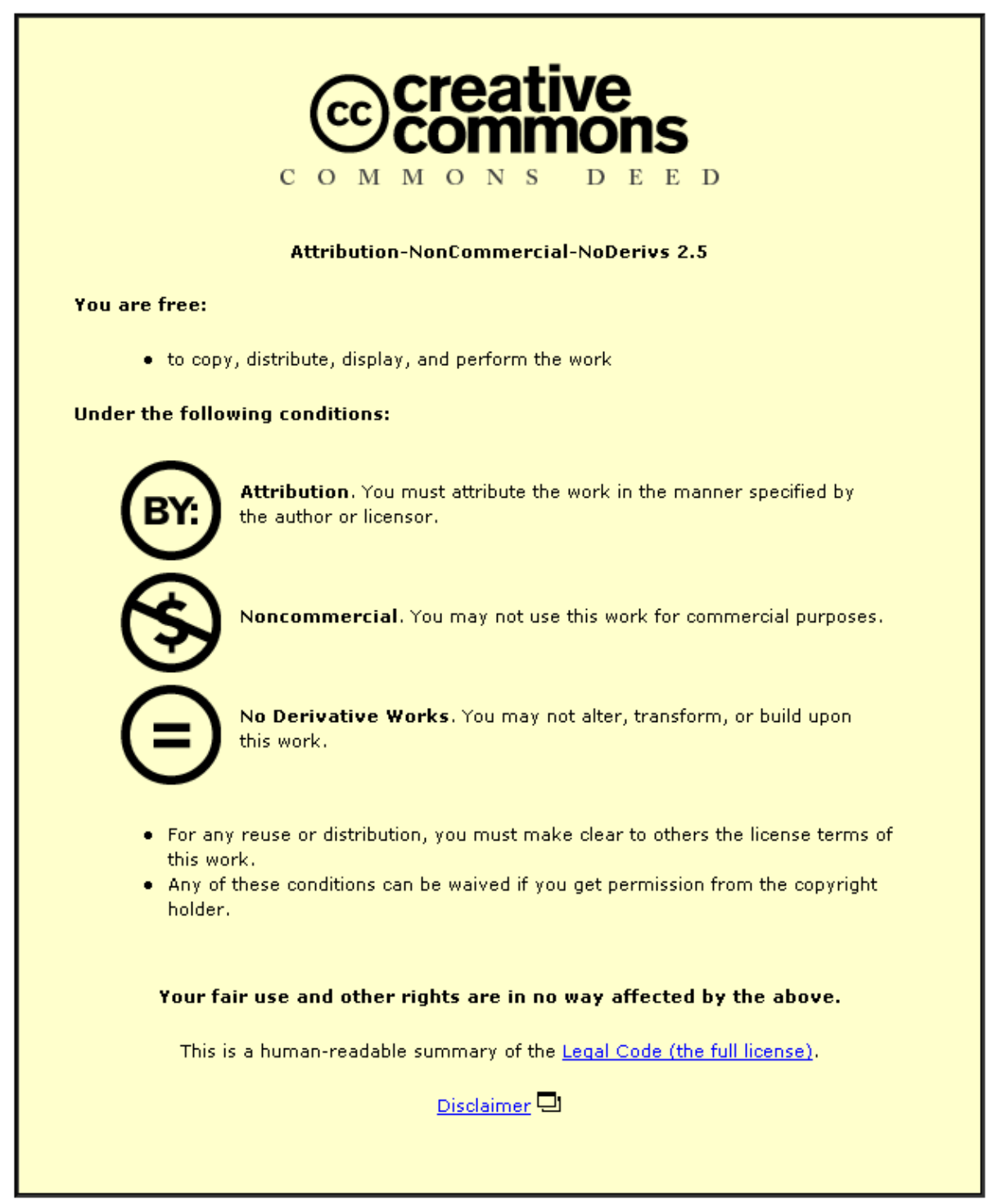

For the full text of this licence, please go to: http://creativecommons.org/licenses/by-nc-nd/2.5/ 


\title{
Modeling the Sputter Deposition of Thin Film Photovoltaics using Long Time Scale Dynamics Techniques
}

\author{
S. Blackwell ${ }^{1}$, R. Smith ${ }^{1}$, S. D. Kenny ${ }^{1}$ and J. M. Walls ${ }^{2}$ \\ ${ }^{1}$ Department of Mathematical Sciences and ${ }^{2}$ Department of Electronic and Electrical \\ Engineering, Loughborough University, Loughborough, Leicestershire, LE11 3TU, UK.
}

\begin{abstract}
Results are presented for modeling the deposition of $\mathrm{Ag}$ and rutile $\mathrm{TiO}_{2}$. The model can be used to examine the effect of varying experimental parameters, such as the substrate bias in the magnetron and the stoichiometry of the deposition species. We illustrate how long time scale dynamics techniques can be used to model the process over experimental time scales. Long time dynamics is achieved through an on-the-fly Kinetic Monte Carlo (otf-KMC) method, which determines diffusion pathways and barriers, in parallel, with no prior knowledge of the involved transitions. Using this otf-KMC method we have modeled the deposition of $\mathrm{Ag}$ and $\mathrm{TiO}_{2}$ for various plasma deposition energies, in the range $1 \mathrm{eV}$ to $100 \mathrm{eV}$. It was found that $\mathrm{Ag}\{111\}$ produces the most crystalline growth when deposited at $40 \mathrm{eV}$. $\mathrm{TiO}_{2}$ growth showed that at energies of $1 \mathrm{eV}$ and $100 \mathrm{eV}$ a porous structure occurs with void formation. At deposition energies of $30 \mathrm{eV}$ and $40 \mathrm{eV}$, a more dense and crystalline rutile growth forms. The results show that deposition energy plays an important role in the resulting thin film quality and surface morphology.
\end{abstract}

\section{INTRODUCTION}

Atomistic simulation has been used as a complementary partner with experiment to address problems in materials science. However, despite advances in computational power, using traditional molecular dynamics (MD) alone to model large systems over long time scales is computationally infeasible. Traditional MD allows simulation for at most a few milliseconds, depending on the system size, whereas the time required to grow one monolayer of silver is 100 milliseconds. Long time scale dynamics combines MD with new techniques, allowing simulation over experimental time scales without biasing the system dynamics. In this work we apply a technique proposed by Henkelman and Jónsson [1]; on-the-fly Kinetic Monte Carlo (otf-KMC). 


\section{THEORY}

Simulations were carried out using the Ackland EAM potential [2] for Ag and a modified variable charge potential for $\mathrm{TiO}_{2}[3,4]$. Periodic boundary conditions were employed for the substrate; with the Coulomb interaction between atoms calculated using the Ewald summation in the case of $\mathrm{TiO}_{2}$.

\section{Molecular Dynamics}

During MD to remove excess energy from the system caused by the deposited ad-unit, a thermostat was attached to layers 2 and 3 of the lattice, while the bottom layer was fixed. Randomly oriented ad-units were deposited normally to the surface, with deposition energies ranging from $1 \mathrm{eV}$ up to $100 \mathrm{eV}$, depending on the simulation.

In order to model the temperature during deposition we heated the lattice to $350 \mathrm{~K}$ before each deposition. MD continued until the lattice returned to this desired temperature and defects stabilized, typically taking between $4-10 \mathrm{ps}$, depending on deposition energy.

\section{On-the-fly Kinetic Monte Carlo}

In traditional Kinetic Monte Carlo (KMC) atoms are assigned to lattice sites and all possible transitions are predefined. This is useful for simple, symmetric crystal structures, however, as systems become more complex traditional KMC cannot perform accurately. With highly defective systems it is possible to miss transitions in the predefined list. otf-KMC aims to rectify this issue by calculating transitions "on-the-fly".

The otf-KMC algorithm involves four fundamental steps, described below:

1. Identify any defects by comparison to a perfect bulk. This produces a defective lattice where defects and their neighboring atoms are included. These are included in the search vector for a transition.

2. Search for possible transitions. Involving only the atoms defined in (1), we use the Relaxation and Translation method (RAT) [4] to locate the saddle points, then, the climbing image Nudged Elastic Band method (NEB) [5] is used to determine the height more accurately, once the transition is found.

3. Calculate the transition rate of each unique transition using the Arrhenius equation (1):

$$
\text { Escape Frequency }=v \exp \left(-E_{b} / k_{B} T\right)
$$

where $v$ is the transition prefactor, $\mathrm{E}_{\mathrm{b}}$ is transition barrier, $\mathrm{k}_{\mathrm{B}}$ is the Boltzmann constant and $\mathrm{T}$ is the temperature (Kelvin). $v$ can be calculated for each transition using the Vineyard [6] method but due to time limitations we normally take the prefactor to be $10^{13}$.

4. Transition searches together with a deposition event are carried out in parallel, on typically 24 cores. A transition or deposition event is chosen from a roulette algorithm that uses this event to evolve the system in time. 


\section{RESULTS AND DISCUSSION}

\section{Ag growth}

The substrate is an Ag $\{111\}$ 900-atom lattice, four layers deep. In the absence of surface defects, monomers and dimers are highly mobile on the surface; low energy depositions produce little damage therefore we begin the simulation with a trimer on the surface, which acts as a nucleation site for the growth. This saves time at the start of the simulation. We ignore transitions with energy barriers above $1 \mathrm{eV}$, as they are very unlikely to occur within the deposition time frame.

The main parameter explored was deposition energy of arriving atoms. Deposition energies from $1 \mathrm{eV}$ to $40 \mathrm{eV}$ were used. Depositing atoms with low arrival energy of $1 \mathrm{eV}$ simulates the evaporation deposition process. Our simulations deposited three monolayers of atoms onto the substrate, at $350 \mathrm{~K}$, over a time of $\sim 0.3$ seconds Figure 1 illustrates the growth of the $\mathrm{Ag}\{111\}$ surface at this energy. Height of atoms is shown using the key, note that the original substrate was 7A high. It is clear that no complete layers have formed; islands form on top of other islands, as atoms do no have enough energy to diffuse down to fill lower layers. We observe a stacking fault in the new growth; the original substrate remained in FCC stacking but there is a stacking fault at the first new layer. Due to the low deposition energy, no mixing occurred between the original and deposited atoms.
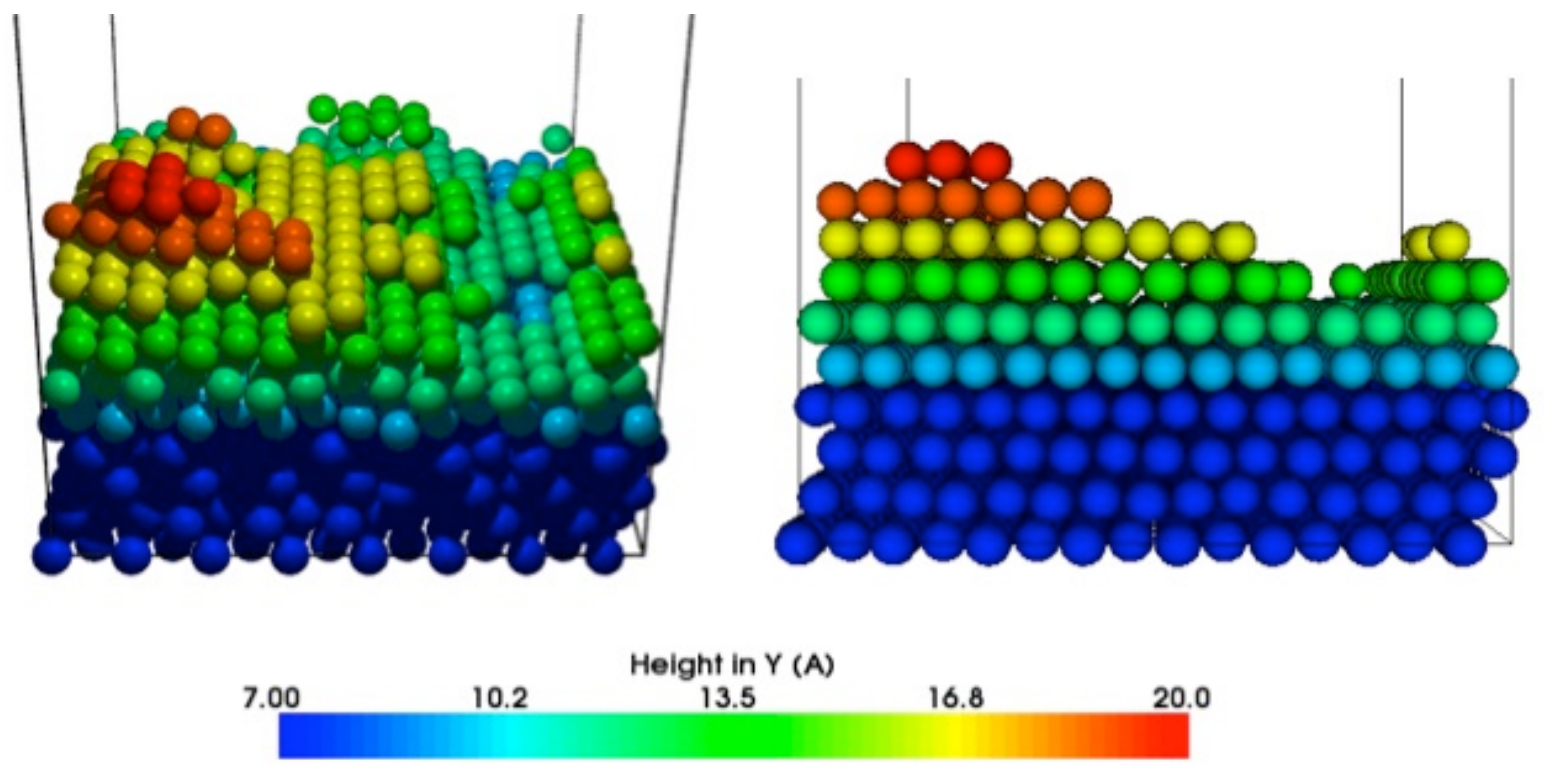

Figure 1. Simulation of the evaporation thin film deposition growth of $\mathrm{Ag}\{111\}$. The low arrival energy of atoms allows little surface diffusion or mixing, resulting in incomplete layers.

Figure 2, simulates the sputter deposition process where atoms arrive with a higher energy of $40 \mathrm{eV}$. After depositing the same number of atoms as above we notice that we have grown two complete new layers, with the third almost complete. This indicates that the increased energy of the arriving atoms allows improved surface diffusion, leading to the completion of lower layers. Arriving atoms now cause damage to the substrate; this damage allows a mixing mechanism whereby damage and repair occurs, which facilitates growth. 


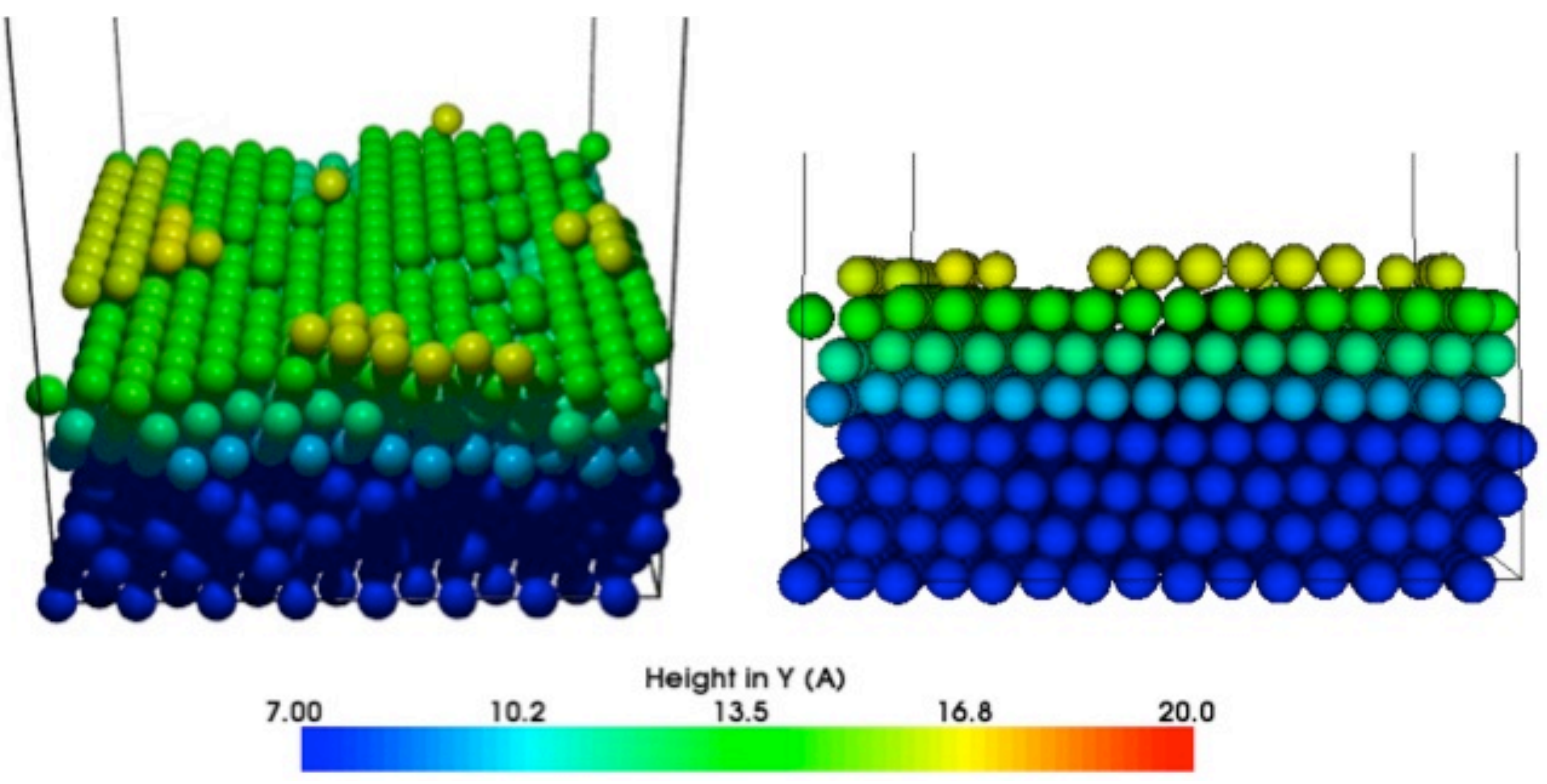

Figure 2. Simulation of the sputter deposition growth of $\mathrm{Ag}\{111\}$. Higher energy Ag deposition allows atoms to be more mobile on the surface, leading to completion of layers, thus producing highly crystalline growth.

\section{Pinhole filling}

Pinholes are an issue in thin film growth; we look into exactly how they are filled. It was found that pinholes are filled by either a direct deposition to a location nearby the pinhole with enough local energy to drop into the pinhole or by a multi-atom concerted motion. Figure 3 illustrates the concerted motion. This concerted motion transition is extremely unlikely to have been included in a traditional lattice KMC model.
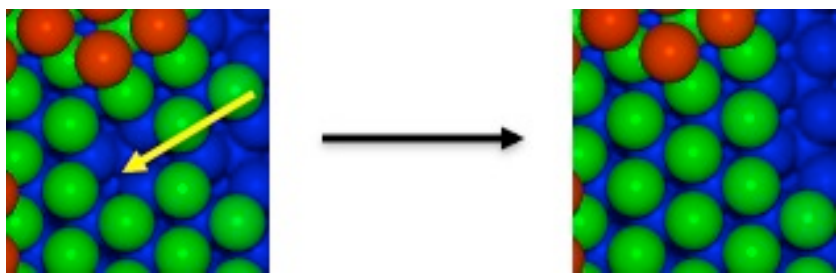

Figure 3. Ag $\{111\}$ surface with a pinhole in the $1^{\text {st }}$ new layer. A concerted motion of two atoms in the same layer; atoms slide across in the direction of the arrow to fill the pinhole, with an associated accessible energy barrier of $0.4 \mathrm{eV}$.

\section{$\underline{\mathrm{TiO}}_{2}$ growth}

We begin the simulation with a smooth rutile lattice eight layers deep, made up of 1536 atoms. Low energy barriers below $0.4 \mathrm{eV}$ are ignored; these were found to be due to atomistic vibrations that did not add to any net diffusion thus could be ignored along with those above 1 $\mathrm{eV}$ to save computational time. When depositing, one of five ad-units is chosen; $\mathrm{O}, \mathrm{O}_{2}, \mathrm{Ti}, \mathrm{TiO}$ or $\mathrm{TiO}_{2}$. These ad-units are deposited at the correct stoichiometry to ensure that $\mathrm{Ti}$ to $\mathrm{O}$ ratio is 
correct at 1:2. Rutile was grown using deposition energies ranging from $1 \mathrm{eV}$ to $100 \mathrm{eV}$. We noticed that at $1 \mathrm{eV}$ and $100 \mathrm{eV}$ the growth produced was highly defective and porous. Whereas the sputter deposition process using a deposition energy of $40 \mathrm{eV}$ produced the most crystalline and dense material. Figure 4 illustrates growth after three monolayers of atoms were deposited, at $40 \mathrm{eV}$ simulating the system for $\sim 4.3$ seconds, with a breakdown of the layers. It is clear than the growth is crystalline with $\mathrm{O}$ atoms preferentially filling the ad-row locations, whereas the $\mathrm{Ti}$ atoms did not. A very important rutile growth mechanism is the drawing out of Ti sub-surface interstitials onto the surface by nearby $\mathrm{O}$ ad-atoms [7].

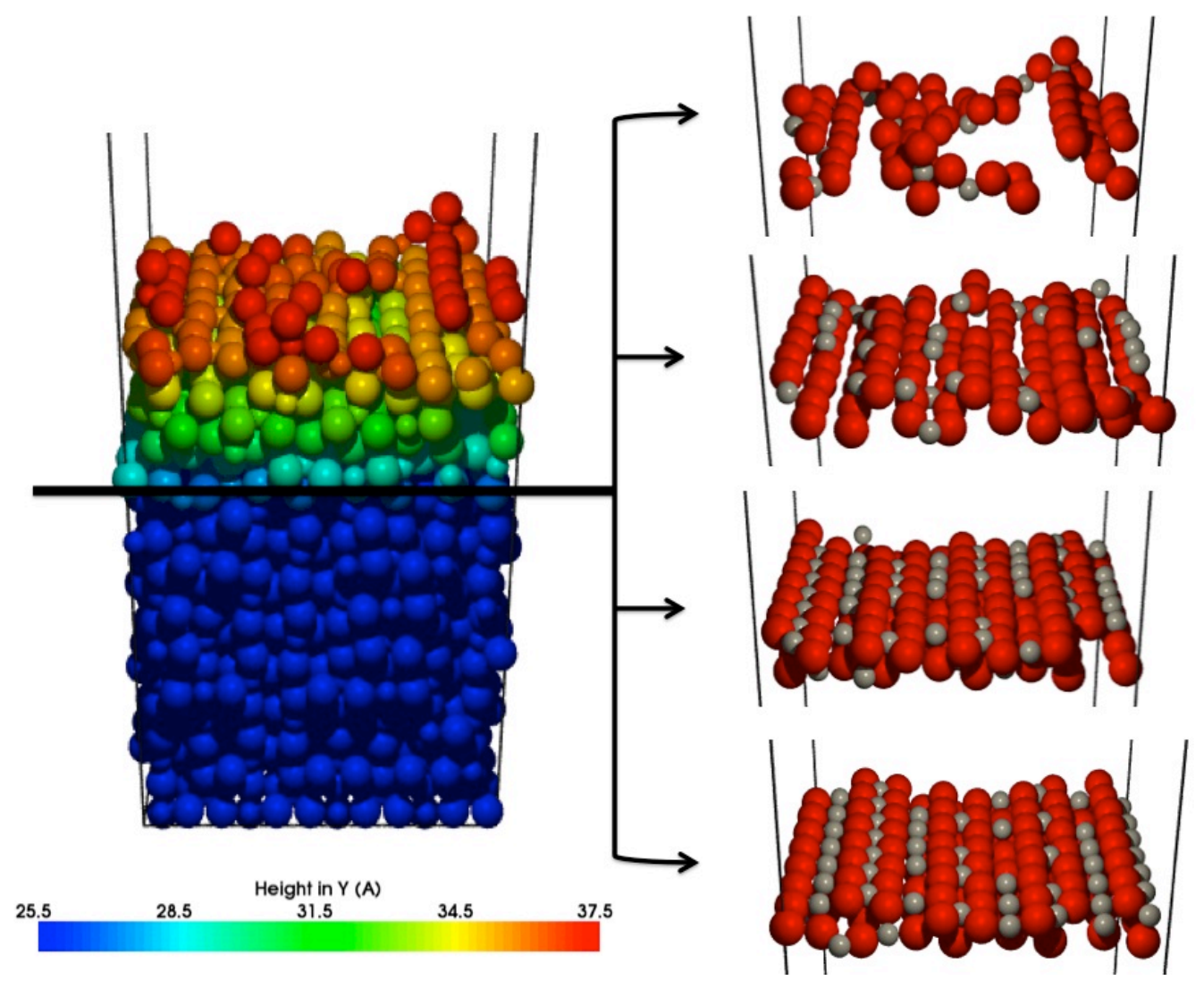

Figure 4. Simulation of sputter deposited growth of $\mathrm{TiO}_{2}$. Smaller sized atoms represent Ti and larger sized atoms represent $\mathrm{O}$. New growth begins at the indicated line, and the new growth has been split into layers. $1^{\text {st }}$ and $2^{\text {nd }}$ grown layers are $\mathrm{O}$ complete, with an excess of $\mathrm{Ti}$.

\section{Stoichiometry}

The stoichiometry of $\mathrm{TiO}_{2}$ is $1: 2$, the final stoichiometry of the original top layer is examined together with the $1^{\text {st }}$ new layer. Figure 5 illustrates that at all deposition energies an excess of $\mathrm{Ti}$ in the top original layer exists, indicating that $\mathrm{Ti}$ interstitials form readily. In the $1^{\text {st }}$ new layer the low and high energy depositions produce incomplete layers, whereas the mid range energies produce complete layers with an excess of Ti interstitials. This fits with the notion of the rutile growth mechanism whereby a high portion of Ti interstitials exist but are drawn out to the surface by $\mathrm{O}$ ad-atoms. 

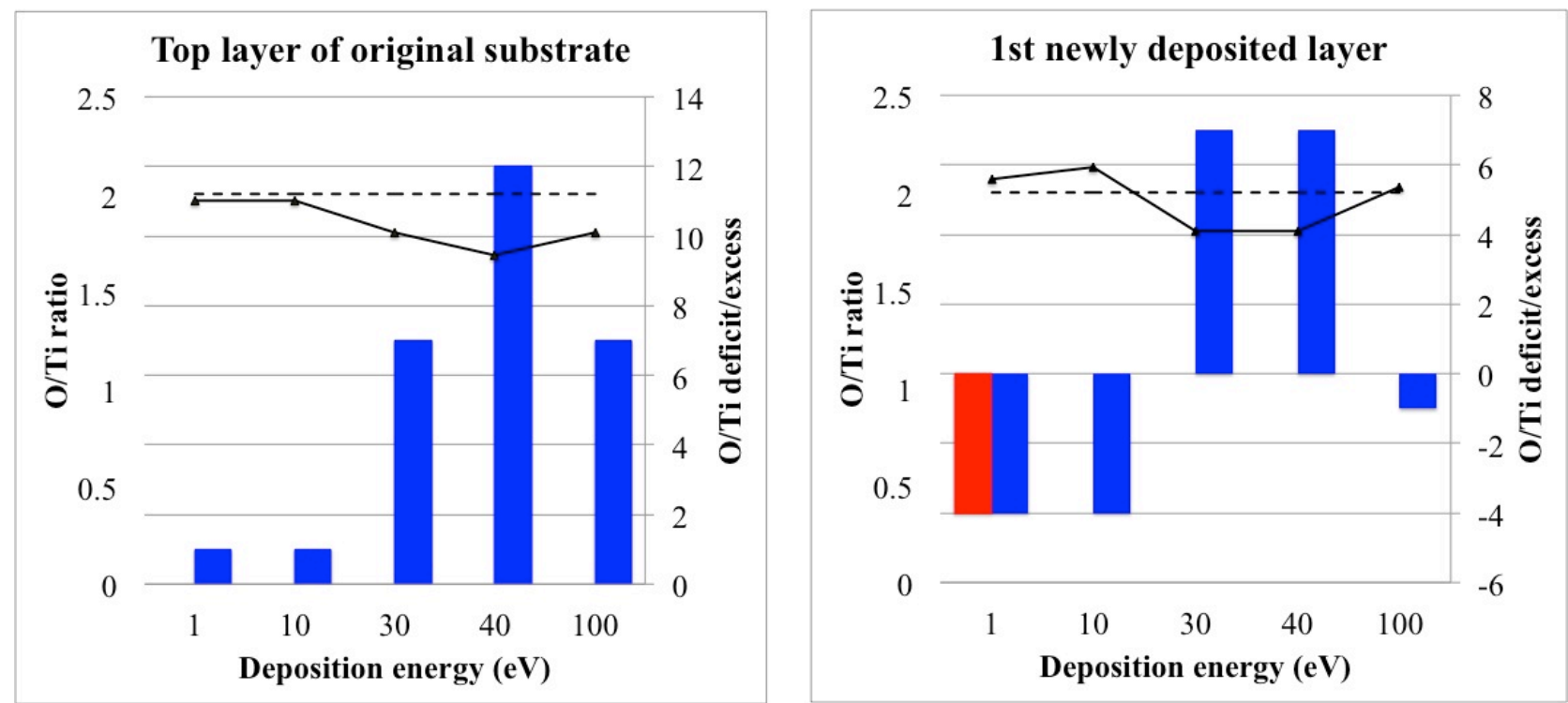

$\square \mathrm{O}$ Deficit/excess $\square \mathrm{Ti}$ Deficit/excess $\rightarrow \mathrm{O} / \mathrm{Ti}$ ratio

Figure 5. Stoichiometry of the top original layer and $1^{\text {st }}$ new layer, noticeable is how $\mathrm{O} / \mathrm{Ti}$ ratio changes due to either excess or deficit of atoms. The dashed line indicates perfect O/Ti ratio.

\section{CONCLUSIONS}

otf-KMC combined with MD allows simulation of growth over realistic time scales. Ag $\{111\}$ produces the most crystalline growth from sputter deposition (deposition energy of 40 $\mathrm{eV}$ ), whereas the lower energy used in evaporation thin film deposition does not allow layers to be completed, thus producing incomplete, void filled structures. Rutile $\mathrm{TiO}_{2}$ shows similar patterns; low and high deposition energies produce incomplete, porous growth whereas the 40 $\mathrm{eV}$ sputter deposition energy produces the most crystalline, dense and complete growth.

\section{REFERENCES}

1. G. Henkelman and H. Jónsson, J. Chem. Phys. 115, 9657 (2001).

2. G. J. Ackland, D. J. Bacon, A. F. Calder, T. Harry, Phil. Mag. A. 75, 713 (1997).

3. A. Hallil, R. Tetot, F. Berthier, I. Braems, and J. Creuze, Phys. Rev. B, 73, 165406 (2006).

4. L. J. Vernon, PhD thesis, Loughborough University, 2010.

5. G. Henkelman, D. Sheppard, and R. Terrell, J. Chem. Phy. 128, 134106 (2008).

6. G. Vineyard, J. Phys. Chem. Solids. 3, 121 (1957).

7. L. J. Vernon, S. D. Kenny, R. Smith and E. Sanville, Phys. Rev. B. 83, 075412 (2011). 